EPJ Web of Conferences 66, 02113 (2014)

DOI: $10.1051 /$ epjconf/ 20146602113

(C) Owned by the authors, published by EDP Sciences, 2014

\title{
Density profiles of light nuclei in Monte Carlo shell-model calcu- lation
}

\author{
T. Yoshida ${ }^{1, a}$, N. Shimizu ${ }^{1}$, T. Abe ${ }^{2}$, and T. Otsuka ${ }^{1,2,3}$ \\ ${ }^{1}$ Center for Nuclear Study, University of Tokyo \\ ${ }^{2}$ Department of Physics, University of Tokyo \\ ${ }^{3}$ National Superconducting Cyclotron Laboratory, Michigan State University, East Lansing, Michigan, USA
}

\begin{abstract}
The wave functions of the ground states for ${ }^{8,10} \mathrm{Be}$ which are obtained from the Monte Carlo shell model (MCSM) are investigated. A method to define an intrinsic state in the MCSM is discussed. The appearance of two- $\alpha$-cluster structure in ${ }^{8} \mathrm{Be}$ and the property of the valence neutrons in ${ }^{10} \mathrm{Be}$ are also discussed.
\end{abstract}

\section{Introduction}

In the large region of the nuclear chart, the shell-model picture is essential to understand the properties of nuclear structure. The conventional shell-model calculations have been performed at the medium mass region. Here, all relevant degrees of freedom of valence nucleons are taken into account, where these particle are on top of an inert core nucleus. For the mass number $A \leq 14$, shell-model calculations without assuming an inert core have been performed. At such light mass region, cluster structure plays an important role. The appearance of $\alpha$ cluster structure has been studied and well confirmed [1].

In this region, the physical values such as electromagnetic and monopole transition strengths are closely related to the cluster configuration of relevant states [2]. Ab initio calculations have recently started for this region. One example is the Green's function Monte Carlo (GFMC) [3] approach. In its analysis of $A=8$ nuclei, the two- $\alpha$-cluster shape emerges $a$ priori from the density distribution. This achievement is important for the shell-model calculation without the inert core. The problem when we use the usual shell-model space is that the single-particle orbits are expanded around the one center of the harmonic oscillator potential. Therefore, to discuss $\alpha$-cluster configuration in view of intrinsic shape has been difficult so far. Recently, the size of the model space which is tractable in the shell-model calculation has been increased due to the development of parallel computers and methodology of shell-model calculations.

When the model space is large enough, it is natural to expect that $\alpha$-cluster configurations are included in the single-particle orbit of the shell model. In fact, the deformation and cluster-like shape for Li isotopes have been investigated by using no core full configuration (NCFC) approach [4]. Another approach is the Monte Carlo shell model (MCSM) [5] method which has been applied to various nuclei by using recent parallel computers with sophisticated energy-minimum search where the conjugate gradient method and energy-variance extrapolation $[6,7]$ are combined. In the proceedings,

ae-mail: yoshida@cns.s.u-tokyo.ac.jp 
we show the density profiles of wave function obtained in the no-core-MCSM calculation. For this purpose, we define the intrinsic state from the no-core-MCSM wave function in the following section. We focus on the ground state of ${ }^{8,10} \mathrm{Be}$ which are expected to have the two- $\alpha$-cluster configuration. In

${ }^{10} \mathrm{Be}$, the density for the valence neutrons is discussed.

\section{Formulation}

In this section, we show how to obtain the intrinsic structure for the no-core-MCSM wave function. In order to investigate the cluster-like state, it is important to extract the intrinsic state from the wave function whose angular momentum is a good quantum number, $J$, in the no-core MCSM. We use a Slater determinant, $\left|\phi_{n}\right\rangle$, to describe a basis state which is defined as

$$
\left|\phi_{n}\right\rangle=\prod_{\alpha=1}^{N_{p}} \sum_{i=1}^{N_{s p}} c_{i}^{\dagger} D_{i \alpha}^{(n)}|-\rangle,
$$

where $|-\rangle$ is a vacuum state and the $c_{i}^{\dagger}$ 's are creation operators on the single-particle orbit, $i$. The resultant wave function is given by a superposition of the angular-momentum-projected Slater determinants,

$$
|\Psi\rangle=P_{M K}^{J^{\pi}}|\Phi\rangle, \quad|\Phi\rangle=\sum_{n=1}^{N_{b}} f_{n}\left|\phi_{n}\right\rangle .
$$

In this study, we use the values, $\pi=+, J=0, M=0$ and $K$-quantum number, $K=0$. The coefficients $f_{n}$ 's are determined by diagonalizing the norm and Hamiltonian matrices, $\left\langle\phi_{i}\left|P^{J^{\pi}}\right| \phi_{j}\right\rangle$ and $\left\langle\phi_{i}\left|H P^{J^{\pi}}\right| \phi_{j}\right\rangle$. In the no-core-MCSM procedure, we tune the value of $D^{(n)}$ for each Slater determinant by the conjugate gradient method in order to minimize the expectation value of the Hamiltonian with $J$-projected wave functions.

In this $J$-projected wave function, cluster-like structure is difficult to be visualized. In order to obtain the intrinsic wave function, we rotate each basis state, following the concept of the GFMC [3]. The intrinsic wave function $\left|\Phi^{\text {intr }}\right\rangle$ is defined as

$$
\left|\Phi^{\mathrm{intr}}\right\rangle \equiv \sum_{n} f_{n} R\left(\Omega_{n}\right)\left|\phi_{n}\right\rangle=\sum_{n} f_{n}\left|\phi_{n}^{R}\right\rangle
$$

with the transformed basis state $\left|\phi_{n}^{R}\right\rangle$. Here, $R\left(\Omega_{n}\right)$ is the rotation operator with an Euler angle $\Omega_{n}$, which is determined by the condition, $\left\langle\phi_{n}^{R}\left|Q_{i, j}\right| \phi_{n}^{R}\right\rangle=\delta_{i, j} q_{i}$, where $Q_{i, j(=x, y, z)}$ are the operators of the mass quadrupole moment. We note that this state, $\left|\Phi^{\text {intr }}\right\rangle$, exactly has the same energy with $|\Phi\rangle$ after the angular momentum projection. It is probable that these aligned basis states have large overlap with each other and make a distinct principal axis toward the $z$-axis when the condition $q_{z}>q_{y}>q_{x}$ is set. The intrinsic density is obtained by the expectation value of the one-body-density operator,

$$
\rho^{\mathrm{intr}}(r)=\left\langle\Phi^{\mathrm{intr}}\left|\sum_{i} \delta\left(r-r_{i}\right)\right| \Phi^{\mathrm{intr}}\right\rangle,
$$

where $r_{i}$ denotes the position of the $i$-th nucleon. We notice that the intrinsic density defined in this section corresponds to the classical picture on the body-fixed frame. Therefore, this definition differs from the one obtained by excluding the $\mathrm{c}$. $\mathrm{m}$. motion from wave function in the laboratory system [4]. 


\section{Result}

In Fig. 1, the proton densities for the $0^{+}$ground state of ${ }^{8} \mathrm{Be}$ are shown. Here, the densities in the laboratory system, which have spherical shape are shown in the left figures. The densities of the wave function $\Phi$ (before alignment) are shown in the middle figures. The intrinsic densities of the wave function $\Phi^{\text {intr }}$ are shown in the right figures.

Here, we use the JISP16 NN interaction which reproduces $N N$ scattering data and properties of deuteron and other light nuclei [8]. As shown in the results of $N_{b}=1$, a clear deformation and its neck structure to be called dumbbell shape appear. We can see that as the number of the Slater determinants $\left(N_{b}\right)$ increases, the densities before the alignment (middle) becomes vaguer because of the mixture of different directions of principal axes of the basis states. On the other hand, the intrinsic densities (right) have clearer dumbbell-like structure for each $N_{b}$. In addition, the density distribution of the intrinsic state is almost unchanged with respect to $N_{b}$. This result indicates the appearance of cluster structure. Since the number of particles for opposite sides of the principal axis are almost the same, this state can be considered as two $\alpha$ clusters. This result is similar to that of the GFMC [3].

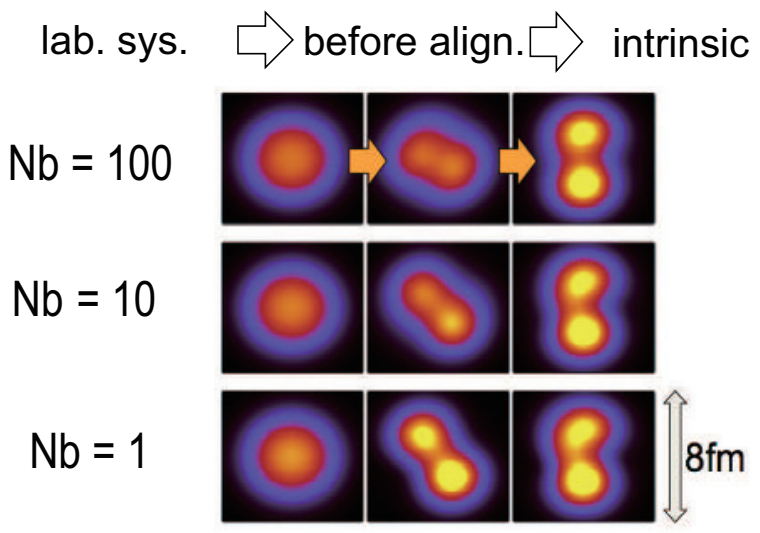

Figure 1. ${ }^{8}$ Be proton densities in the laboratory system with $J$-projected states, $|\Psi\rangle$, (left) and before alignment of principal axes of unprojected basis states, $|\Phi\rangle$, (middle) and of intrinsic state, $\left|\Phi^{\text {intr }}\right\rangle$, (right) for various $N_{b}$ and slices along $y z$ plane. The numbers of basis states are $N_{b}=1,10$ and 100 for the lower, middle and upper figures, respectively. Slices along $y z$ plane are $x=0 \mathrm{fm}$ plane for each figure. The size of each box is $8 \mathrm{fm} \times 8 \mathrm{fm}$. The model space and harmonic oscillator energy are $N_{\text {shell }}=4$ and $\hbar \omega=20 \mathrm{MeV}$, respectively.

We perform the same analysis for the $0^{+}$ground state of ${ }^{10} \mathrm{Be}$. Here, we use the parameters for MCSM, $N_{\text {shell }}=4$ and $\hbar \omega=25 \mathrm{MeV}$, respectively. The wave function is determined by the conjugate gradient method, where the number of basis states, $N_{b}=100$. We depict the densities as shown in Fig. 2 . The valence-neutron density is estimated by subtracting the proton density from the neutron density. We find the ${ }^{8} \mathrm{Be}$-like core in the matter density which has two $\alpha$ cluster structure. We see that the valence neutrons are located mainly around the neck part of ${ }^{8} \mathrm{Be}$-like core. This picture is consistent with the behavior of $\pi$-orbit in the molecular orbital model of ${ }^{10} \mathrm{Be}$ [9]. 

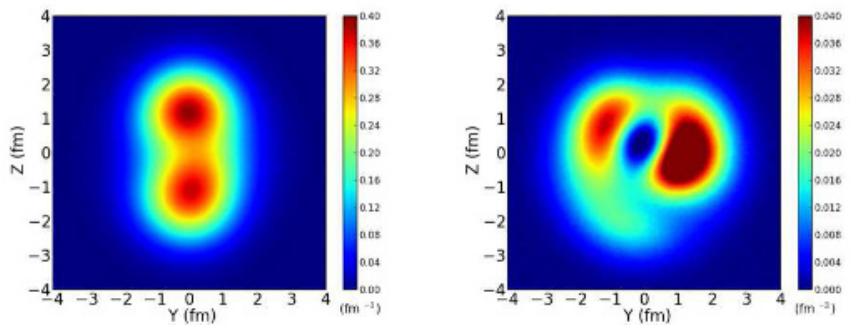

Figure 2. The densities of the $0^{+}$ground state for ${ }^{10} \mathrm{Be}$ are shown. The number of basis states is $N_{b}=100$. The model space and harmonic oscillator energy are $N_{\text {shell }}=4$ and $\hbar \omega=25 \mathrm{MeV}$, respectively. The intrinsic densities for matter and valence neutrons are shown (left and right figures, respectively).

\section{Summary}

We use the method by which one can extract the intrinsic structure from the wave function in the nocore MCSM. We show that the neck shape indicating the two $\alpha$ clusters emerges within a relatively small number of the harmonic oscillator quanta. We notice that the shape which appears in the nocore MCSM has the similar property in the GFMC in terms of the density for the $0^{+}$ground state of ${ }^{8} \mathrm{Be}$. In the analysis for the $0^{+}$ground state of ${ }^{10} \mathrm{Be}$, we see the two- $\alpha$-cluster shape and $\pi$-orbit-like configuration of the valence neutrons. This corresponds to the result of the $\alpha$ cluster model.

\section{Acknowledgments}

We would like to thank Prof. Pieter Maris, Prof. James P. Vary and Prof. Yutaka Utsuno for fruitful discussions. This work has been supported by the SPIRE Field 5 from MEXT, and the CNS-RIKEN joint project for large-scale nuclear structure calculations. The numerical calculations were performed on the T2K Open Supercomputers at the University of Tokyo and Tsukuba University.

\section{References}

[1] Y. Fujiwara, H. Horiuchi, K. Ikeda, M. Kamimura, K. Katō,Y. Suzuki and E. Uegaki, Prog. Theor. Phys. Suppl. 68, 29 (1980).

[2] T. Yamada, Y. Funaki, H. Horiuchi, K. Ikeda and A. Tohsaki, Prog. Theor. Phys. 120, 6 (2008).

[3] R. B. Wiringa, S. C. Pieper, J. Carlson and V. R. Pandharipande, Phys. Rev. C 62, 014001 (2000).

[4] C. Cockrell, J. P. Vary and P. Maris, Phys. Rev. C 86, 034325 (2012).

[5] T. Abe, P. Maris, T. Otsuka, N. Shimizu, Y. Utsuno and J. P. Vary, Phys. Rev. C 86, 054301 (2012).

[6] T. Otsuka, M. Honma, T. Mizusaki, N. Shimizu and Y. Utsuno, Prog. Part. Nucl. Phys. 47, 319 (2001).

[7] N. Shimizu, T. Abe, Y. Tsunoda, Y. Utsuno, T. Yoshida, T. Mizusaki, M. Honma and T. Otsuka, Prog. Theor. Exp. Phys. 2012, 01A205 (2012).

[8] A. M. Shirokov, J. P. Vary, A. I. Mazur, S. A. Zaytsev and T. A. Weber, Phys. Lett. B 621, 96 (2005).

[9] N. Itagaki and S. Okabe, Phys. Rev. C 61, 044306 (2000). 